\title{
Breast conservation therapy without capsular contracture in young augmented women using interstitial brachytherapy
}

\author{
Robert Kuske, MD \\ Virginia G. Piper Cancer Center, Scottsdale Healthcare Shea, Scottsdale, Arizona, USA
}

\begin{abstract}
Purpose: To describe a breast-conserving technique using interstitial brachytherapy after lumpectomy and axillary nodal sampling in selected women who are diagnosed with breast cancer in the presence of augmentation mammoplasty.

Material and methods: Over the past 20 years, we have developed and improved a technique of "pinch view" imageguided catheter insertion that avoids implant puncture. Selection criteria include: 1) women of any age with either subpectoral or retroglandular, augmentation implants (silicone or saline) who were diagnosed with stages Tis, T1, T2, N0, or N1 breast cancer; 2) any pathologic subtype of malignant breast cancer was accepted; 3) microscopic tumor extent $\leq 3 \mathrm{~cm} ; 4$ ) axillary node negative or metastasis to 1 to 3 nodes without extracapsular extension; and 5) surgical margins clear by the NSABP "no ink on tumor" definition. More than 250 women have been successfully treated. Patients were treated with high dose rate Iridium-192 brachytherapy to 34 Gy in 10 or 32 Gy in 8 twice daily fractions. The target volume was the surgical cavity edge with 1.5 to $2 \mathrm{~cm}$ margin using 3-D treatment planning systems.

Results: The implant technique as currently employed is described. There have been no implant ruptures, and the Planning Treatment Volume (PTV-eval) exhibited at least $90 \%$ coverage by the $90 \%$ isodose line in the vast majority of cases. Dose Homogeneity Index exceeded 70\% in most cases. The maximum skin dose was below the prescription dose in every case. Other than some patients with pre-existing capsular contracture, less than $5 \%$ experience new capsular contracture after interstitial brachytherapy.

Conclusions: A technique of reliable and reproducible accelerated partial breast irradiation is described that minimizes the risk of capsular contracture by avoiding circumferential dose to the foreign body in the breast.
\end{abstract}

Key words: accelerated partial breast irradiation, brachytherapy, breast augmentation, breast cancer treatment.

\section{Purpose}

The number of women who are diagnosed with breast cancer in the presence of breast augmentation is dramatically increasing each year (Fig. 1). For the past 20 years, breast augmentation has been one of the most common surgical procedures in the United State, Brazil, and other countries [1]. Women who had their implants a decade or more ago at young ages are now at the age when their risk of breast cancer is significant. Oncologists are seeing more and more of these patients, and there is little in the literature about how to treat them. Indeed, the surgical literature suggests mastectomy and reconstruction as the preferred treatment, because of the reported $55 \%$ or higher rate of significant capsular contracture after whole breast external beam radiotherapy [2,3]. Over the past 20 years, the author has treated 250 women after lumpectomy and axillary dissection or sentinel node biopsy with interstitial brachytherapy to complete breast conservation therapy. This technique is known as accelerated partial breast irradiation (APBI), a 4 or 5-day alternative to 6 or 7 -week whole breast irradiation (WBI). The advantage is reducing radiation exposure to only the affected part of the breast in the vicinity of the original tumor site, thereby minimizing dose to the lung, heart, chest wall, ribs, and normal breast or nodal tissue. In women with augmentation, the radiation dose cloud does not encompass the entire implant, eliminating circumferential fibrosis that causes capsular contracture. This technique is described in this manuscript for the first time.

\section{Material and methods}

More than 250 women with breast augmentation and select breast cancers have been treated over the past 20 years 


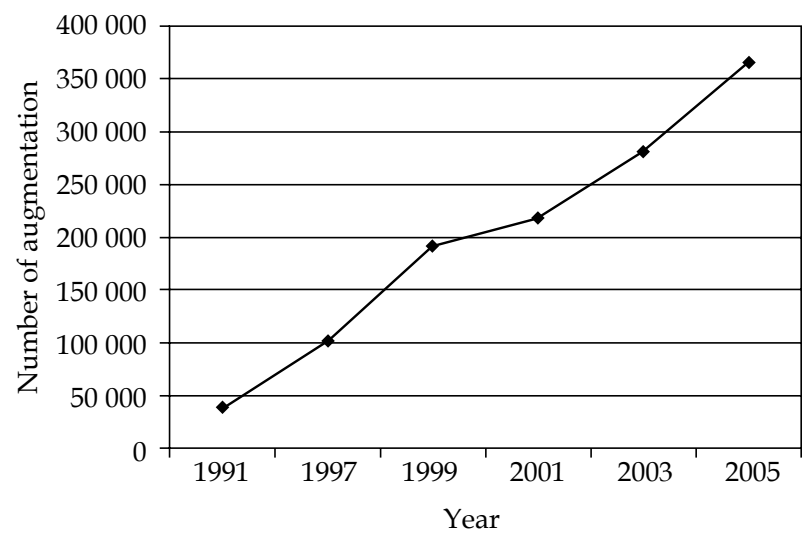

Fig. 1. Rate of breast augmentation over time

with interstitial brachytherapy. Selection criteria have been consistent and uniform, with tumors less than or equal to $3 \mathrm{~cm}$ that have been excised with clear margins (no ink on tumor), and zero to three axillary nodes positive without extracapsular extension accepted. The technique of image-guided interstitial brachytherapy catheter insertion is described herein. An optional breast ultrasound is performed on the day of consultation in the radiation oncology office. When a seroma is clearly seen, the radiation oncologist can visualize the lumpectomy cavity in 3 dimensions, allowing pre-planning. With oncoplastic technique, which is a fusion of breast cancer surgery with plastic surgical principles, the cavity is usually closed when the tissue is re-shaped and approximated. With oncoplasty, pre-procedure ultrasound is rarely helpful, so a CT in radiation oncology on the day of the procedure defines the cavity. No general anesthesia or conscious sedation is necessary. One hour before the procedure, the patient takes a narcotic (eg. Nucynta, Percocet, or Vicodin) and an anxiolytic/muscle relaxer (eg. Valium or Lorazepam) (Table 1).

Table 1. Local anesthetic formulae and pre-operative medications

Local anesthetic formulae and pre-operative medications

Skin wheal $\quad 35 \mathrm{cc} 1 \%$ lidocaine with epinephrine

2 cc $8.4 \%$ sodium bicarbonate

35 cc lactated Ringer's solution

Tumescent 25 cc $2 \%$ lidocaine with epinephrine

3 cc $8.4 \%$ sodium bicarbonate

215 cc lactated Ringer's solution

mEq sodium bicarbonate

Average amount injected per case

30 cc skin wheal mixture through a $27 \mathrm{G}$ needle, 1.25 inches long with a 10 cc syringe, on each side of the template

150 cc tumescent mixture through a $25 \mathrm{G}$ needle, 1.5 inches long with a $10 \mathrm{cc}$ syringe, on each side of the template

Pre-op meds

Nucynta, $50 \mathrm{mg}$ taken p.o. 1 hour before

Valium, $5 \mathrm{mg}$ taken p.o. 1 hour before topical EMLA cream, $30 \mathrm{gm}$ applied under plastic wrap 1 hour before the procedure
The patient is positioned supine or partly decubitus on the CT table in radiation oncology, usually with the ipsilateral arm comfortably at her side. A wide Betadine skin prep covers the entire breast. Sterile towels and drapes surround the breast. The breast is massaged to loosen it off the augmentation implant, and palpation of the lumpectomy site with the ultrasound or CT images in mind help plan template orientation. The breast tissue at the lumpectomy site must be bunched up while the implant is pushed in the reverse direction, similar to Eklund or "pinch view" or "push back" mammography that is standard imaging for women with implants. Note that the thickness of the breast tissue may be only $1.5 \mathrm{~cm}$ in the relaxed state before pinching, but the thickness increases to 3 to $4 \mathrm{~cm}$ after bunching. With one hand, the breast is strongly lifted up and away from the augmentation implant, and the implant pushed backward, while the breast brachytherapy template is positioned (Fig. 2). The front and back plates are closed with moderate compression, so that the deep plane is as close to the implant as feasible. Side clamps are attached to the template to make the plates parallel. The surgical assistant holds the template against the augmentation to prevent slippage. The radiation oncologist chooses 3 holes for anchoring needles that are shallow and safely away from the implant to avoid puncture. These holes are chosen to be likely in the target volume. A skin wheal is raised on both the proximal and distal sides with half-strength buffered local anesthetic, and dilute tumescent buffered local anesthetic is injected along the chosen pathway (Table 1). Thin $27 \mathrm{G}$ needles are used for the skin wheal, and a $25 \mathrm{G}$ for the tumescent. The three anchoring needles are inserted, with care to ensure that they remain perpendicular to the plane of the template as they traverse the breast, exiting the hole on the back template.

After the 3 anchoring needles, the template will not slip and will maintain its position relative to the augmentation implant. A breast CT without contrast is obtained while the template is attached. These images are sent by Ethernet to the computer with 3-d brachytherapy software. Image-guidance is a key to the success of this procedure. On each slice, the medical physicist or dosimetrist, under the supervision of the radiation oncologist, contours the implant, skin, and lumpectomy edges. The software then expands the cavity volume by 1.5 to $2 \mathrm{~cm}$ in all directions, except for $5 \mathrm{~mm}$ from the implant and $5 \mathrm{~mm}$ from the skin surface, creating a modified planning treatment volume (PTV-eval). A 3-dimensional rendition of the breast is rendered by the software, and these images are rotated until the front and back template holes are aligned. The physicist then outlines the maximum cross-sectional area of the PTV-eval. The physicist also outlines the surface of the implant and the skin, so that they are displayed on the "needles eye view" when the templates are aligned. The CT slices are then scrolled until the large holes on the back template are in view.

With the PTV-eval, skin and implant displayed (Fig. 3), it is simple to choose coordinates of template holes that are within or just beyond the PTV-eval, but do not intersect with the implant $+5 \mathrm{~mm}$ or the go outside the skin surface (Fig. 4). Skin wheal and tumescent local anesthet- 

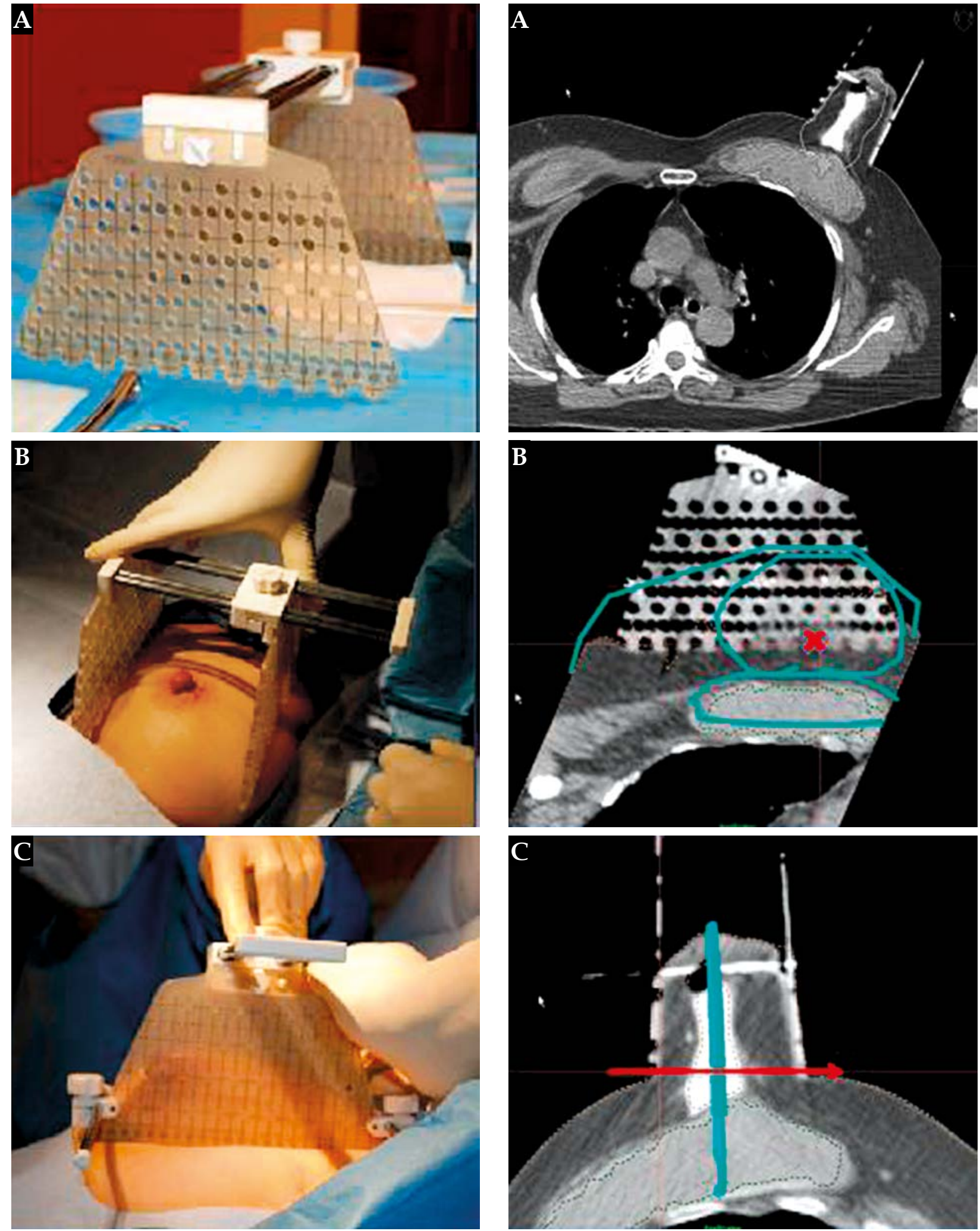

Fig. 2. The breast brachytherapy template

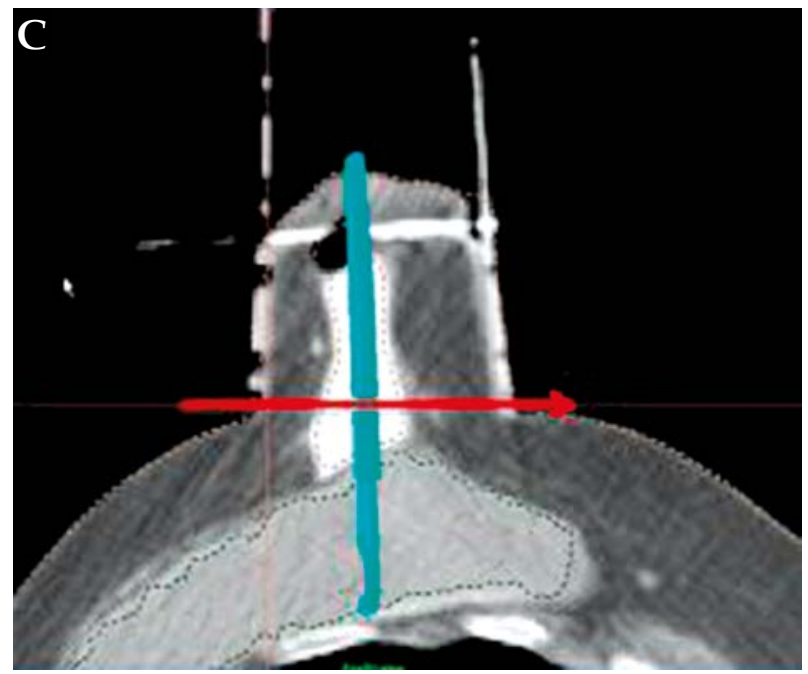

Fig. 3. Image-guided needle insertion with the implant, skin, PTV-eval, and holes of the template displayed

For your first few procedures, you may wish to obtain ic as described above are then injected. $19 \mathrm{G}$ brachytherapy needles are then inserted perfectly perpendicular to the plane of the template. a CT at this point to prove to yourself that the needles have not intersected with the implant. The template is 


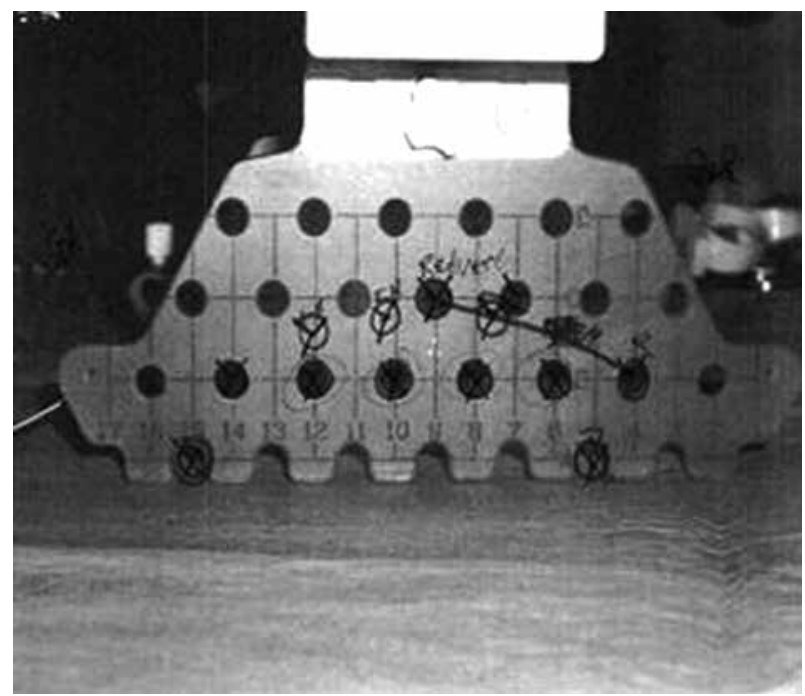

Fig. 4. Diagram of the template with the chosen holes marked

then disassembled and only the needles remain in place with the breast relaxed from compression. Additional freehand needles can be inserted to cover any gaps in coverage, if necessary, being careful to manually push the implant away from the needle's pathway, and keep the needles parallel. Polyethylene catheters are then inserted into each needle, and pulled until the needle is out and the distal button is flush with the skin. A button is placed on the proximal end and positioned against the skin. The catheter is then trimmed. The patient then goes to lunch with her significant other, and returns 2 hours later, after any swelling has dissipated, for the treatment planning CT from which the dwell times and dose cloud shaping is performed. Physics is given 24 hours to perfect the dose plan, so treatment commences on day 2 after the insertion. This procedure is typically painless and bloodless. The local anesthetic lasts approximately 6 hours after the insertion, and the included epinephrine prevents blood loss. Patients usually require pain medication for the first 12-24 hours after the procedure, however none thereafter. Prophylactic antibiotics are not routinely prescribed, and less than $20 \%$ receive antibiotics later because of fever, chills, unusual erythema, or other signs of infection.

Certain dosimetric guidelines are routine. The prescription isodose line can touch the skin surface, but not extend beyond it. We strive for 95\% coverage of the PTV-eval by the $95 \%$ isodose. The dose homogeneity index is usually $10 \%$ less than what we see with non-augmented breasts $75-80 \%$, but rarely below $70 \%$. The $150 \%$ isodose lines may kiss, but not coalesce. If the brachytherapy does not meet these guidelines, additional needles are inserted under local anesthetic to improve coverage and homogeneity.

\section{Results}

The average number of catheters in augmented breasts is 19 , in contrast to 22 catheters in non-augmented breasts, for a $2 \mathrm{~cm}$ growth of the cavity volume. No implants have been punctured, and catheter insertion was successfully accomplished in all augmented women. The mean coverage of the PTV-eval by the $90 \%$ isodose line was $96 \%$. The mean dose homogeneity index is $80 \%$. The brachytherapy entry and exit pockmarks become invisible in $90 \%$ of patients by one year after treatment. Cosmetic outcomes are good/excellent in more than $90 \%$ of patients treated. $10 \%$ of patients are noted to have Baker 2 to 4 capsular contracture at the time of consultation before treatment of her breast cancer. Less than $5 \%$ experience new or additional capsular contracture after interstitial brachytherapy.

Tumor control, survival rates, cosmetic outcomes, and toxicities other than capsular contracture will be reported in another publication.

\section{Discussion}

In addition to very low lung and heart doses, and the advantages to busy women of a shorter treatment time, reduction in the high rate of capsular contracture noted by other investigators is paramount to this study. Capsular contracture is spherical fibrosis caused by circumferential

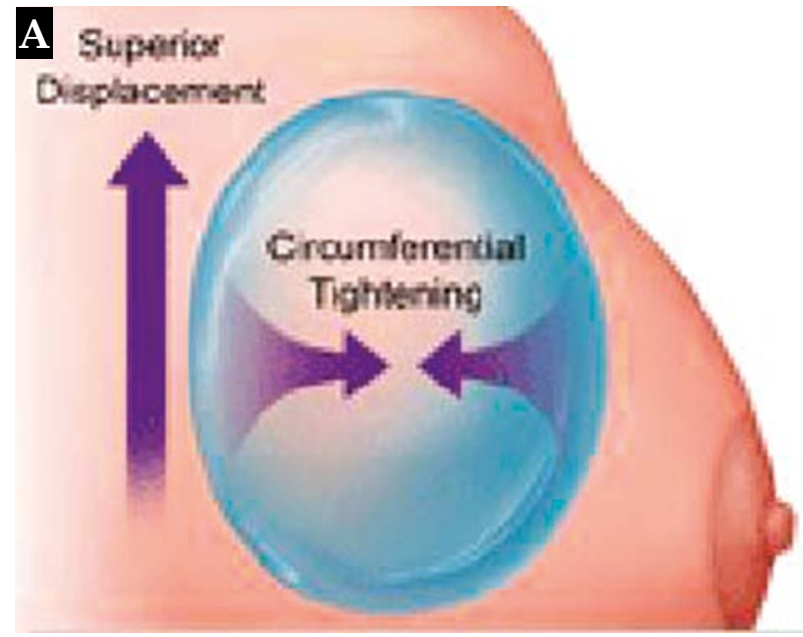

Fig. 5. Spherical fibrosis, capsular contracture

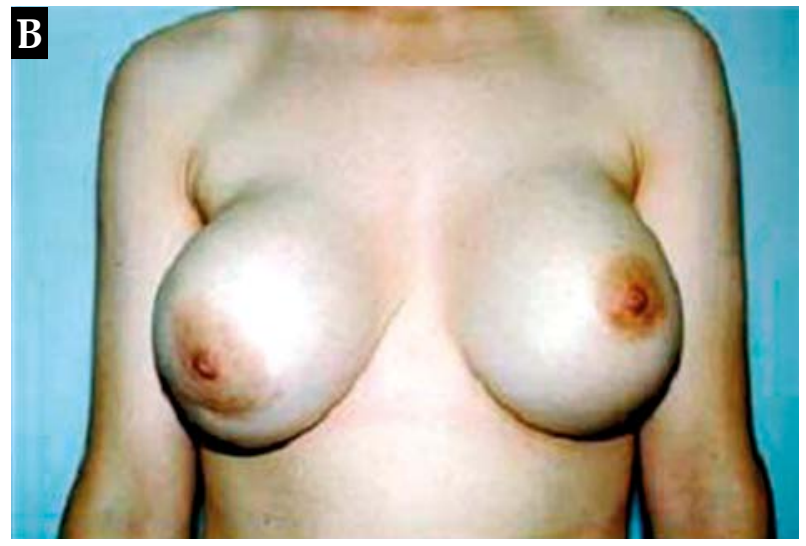


exposure of the foreign body implant by whole breast irradiation (Fig. 5). Patients know this as "painful hardening of the implant". It can be seen without radiotherapy as well, and is a known risk of breast augmentation.

Neil Handel and Melvin Silverstein reported a capsular contracture rate of $55 \%$ after whole breast irradiation in the presence of breast augmentation $[2,3]$. This high rate of capsular contracture has led many surgeons to recommend mastectomy followed by reconstruction as the preferred treatment for early stage breast cancer in women with implants. This recommendation is disappointing to most of these women, as they have already declared their priority on cosmesis by the fact that they have had augmentation in the first place.

Without implants, breast conservation therapy is considered the preferred method of early-stage breast cancer treatment, because survival rates are the same as mastectomy and it allows women to remain whole [4]. Stage for stage, breast conservation should be offered to augmented women as well. The scars are not as long, the surgery takes less time and is less expensive, and the breast remains sensate. Preventing capsular contracture is key to expanding breast conservation in this growing population of women. This goal was the focus of this 20-year investigation into a technique that minimizes the risk of symptomatic capsular contracture.

In many cancer types treated in radiation oncology, image-guidance and tighter conformal radiation fields have improved tumor control rates and decreased toxicity [5]. Breast brachytherapy is an example of an advance in dose-delivery that conforms precisely to the target volume, minimizing exposure of normal tissues. The rapid dose fall-off, inherent in the physics of brachytherapy, is especially attractive in the treatment of women with augmented breasts. The prescription dose is at the periphery, and tissue inside that dose envelope is $~ 15 \%$ higher dose, so the dosimetry follows the biology of breast cancer cell density gradient. Since only a small surface area of the implant is exposed to ionizing radiation, scar tissue does not envelope the silicone or saline implant. The risk of capsular contracture is significantly reduced.

\section{Conclusions}

The concept of inserting sharp local anesthetic and brachytherapy needles close to the implant, without puncturing it, is intimidating to many radiation oncologists. For this reason, a "pinch view" image-guided technique utilizing a template and 3-d treatment-planning software has been developed and is described in this manuscript. As described, the method is simple, safe, and reproducible from one institution to the next. Long-term data [6] supports the conclusion that interstitial breast brachytherapy may be the treatment of choice for select women with early stage breast cancer in the presence of breast augmentation.

\section{Acknowledgements}

The author would like to acknowledge the efforts of Rezwan Chowdhury, MD, a former brachytherapy fellow, who now practices in British Columbia, Canada. Kristina Allen and Carrie Farkas are noted for their administrative assistance for these patients. Margaret Snyder, RN, collected and analyzed the data. The breast surgeons contributed their patients and support of the program.

\section{Disclosure}

Author reports no conflicts of interest.

\section{References}

1. International Society of Aesthetic Plastic Surgery. ISAPS international survey on aesthetic/cosmetic procedures performed in 2011. Available at: http://www.isaps.org/files/ html-contents/Downloads/ISAPS\%20Results \%20-\%20Procedures\%20in\%202011.pdf

2. Handel N. The Effect of Silicone implants on the diagnosis, prognosis, and treatment of breast cancer. Plast Reconstr Surg 2007; 120 (7 Suppl 1): 81S-93S.

3. Handel N, Silverstein MJ. Breast cancer diagnosis and prognosis in augmented women. Plast Reconstr Surg 2006; 118: 587-596.

4. NIH Consensus Conference: Treatment of early-stage breast cancer. JAMA 1991; 265: 391.

5. Dawson LA, Sharpe MB. Image-guided radiotherapy: rationale, benefits, and limitations. Lancet Oncol 2006; 7: 848-858.

6. Kuske R, Chowdhury R, Snyder M et al. Accelerated Partial Breast Irradiation for Select Breast Cancers in the Presence of Augmentation. Int J Radiat Oncol Biol Phys 2012; 84: S87. 